University of New Orleans

ScholarWorks@UNO

$12-1-1986$

\title{
Polarizing beam splitters for infrared and millimeter waves using single-layer-coated dielectric slab or unbacked films
}

\author{
R. M.A. Azzam \\ University of New Orleans, razzam@uno.edu
}

Follow this and additional works at: https://scholarworks.uno.edu/ee_facpubs

Part of the Electrical and Electronics Commons, and the Optics Commons

\section{Recommended Citation}

R. M. A. Azzam, "Polarizing beam splitters for infrared and millimeter waves using single-layer-coated dielectric slab or unbacked films," Appl. Opt. 25, 4225-4227 (1986)

This Article is brought to you for free and open access by the Department of Electrical Engineering at ScholarWorks@UNO. It has been accepted for inclusion in Electrical Engineering Faculty Publications by an authorized administrator of ScholarWorks@UNO. For more information, please contact scholarworks@uno.edu. 


$$
\begin{aligned}
\alpha & =\widetilde{R} \cos 2 \psi /(1-\bar{R}), \\
s_{1} & =S_{1} / S_{0}, \quad s_{2}=S_{2} / S_{0} .
\end{aligned}
$$

Digital Fourier analysis of $i_{d}$ determines the cosine and sine amplitudes $a$ and $b$, and from Eqs. (11) one gets the normalized Stokes parameters

$$
s_{1}=a / \alpha, \quad s_{2}=b / \alpha .
$$

The single important parameter $\alpha$ can be determined in a simple calibration step in which RODE is illuminated by totally linearly polarized light of arbitrary azimuth (by placing a good linear polarizer in the path of the incident beam), and the corresponding Fourier amplitudes $a_{c}$ and $b_{c}$ of the output calibration signal are recorded. Then we can use the fact that

$$
s_{1}^{2}+s_{2}^{2}=1
$$

for total incident linear polarization to obtain, using Eqs. (14),

$$
\alpha=\left(a_{c}^{2}+b_{c}^{2}\right)^{1 / 2} .
$$

The calibration signal can be put in the form $i=i_{0}[1+\alpha$ $\left.\cos \left(2 \theta-2 \theta_{0}\right)\right]$, where $\theta_{0}$ is the azimuth of the incident linear vibration from the reference plane and $\alpha$ represents the modulation depth. This identifies $\alpha$ with the parameter $m_{L}$ of Ref. 1. This can also be verified by manipulating Eq. (12) as follows. Let $R_{p}$ and $R_{s}$ be the detector surface reflectances for incident $p$ - and $s$-polarized light. Then we can write

$$
\begin{aligned}
\bar{R} & =1 / 2\left(R_{p}+R_{s}\right), \\
\cos 2 \psi & =\left(1-\tan ^{2} \psi\right) /\left(1+\tan ^{2} \psi\right) \\
& =\left[1-\left(R_{p} / R_{s}\right)\right] /\left[1+\left(R_{p} / R_{s}\right)\right] \\
& =\left(R_{s}-R_{p}\right) /\left(R_{s}+R_{p}\right) .
\end{aligned}
$$

If $\bar{R}$ and $\cos 2 \psi$ in Eq. (12) are replaced by their values from Eqs. (17), one gets

$$
\begin{aligned}
\alpha & =\left(R_{s}-R_{p}\right) /\left(2-R_{s}-R_{p}\right) \\
& =m_{L} .
\end{aligned}
$$

It should be noted that $\alpha$ is a function of wavelength ${ }^{1}$ and must be measured over the useful spectral range of the detector for spectroscopic applications.

To summarize: we have shown that the rotating-detector ellipsometer (RODE), first described in Ref. 1, can be used to measure the first three Stokes parameters of incident light which is in a general state of partial polarization. This considerably widens the scope of possible applications of this unique and simple instrument.

The author sincerely appreciates the kind hospitality of the Département de Physique du Solide, Université de Provence, Marseille, France, where this work was done. A Fulbright Senior Research Scholar Award is also gratefully acknowledged.

\section{References}

1. R. M. A. Azzam, "Rotating-Detector Ellipsometer for Measurement of the State of Polarization of Light," Opt. Lett. 10, 427 (1985).

2. T. Geherls, Ed., Planets, Stars and Nebulae Studied with Photopolarimetry (U. Arizona Press, Tucson, 1974).
3. D. L. Coffeen, "Polarization and Scattering Characteristics in the Atmospheres of Earth, Venus, and Jupiter," J. Opt. Soc. Am. 69, 1051 (1979).

4. T. Prosch, D. Hennings, and E. Raschke, "Video Polarimetry: A New Imaging Technique in Atmospheric Science," Appl. Opt. 22, 1360 (1983).

5. R. N. A. Azzam and N. M. Bashara, Ellipsometry and Polarized Ligh: (North-Holland, Amsterdam, 1977), p. 491.

6. P.S. Hauge, "Survey of Methods for the Complete Determination of the State of Polarization," Proc. Soc. Photo-Opt. Instrum. Eng. 88,3 (1976).

\section{Polarizing beam splitters for infrared and millimeter waves using single-layer-coated dielectric slab or unbacked films}

\section{R. M. A. Azzam}

University of New Orleans, Department of Electrical Engineering, Lakefront, New Orleans, Louisiana 70148. Received 16 June 1986. 0003-6935/86/234225-03\$02.00/0.

(C) 1986 Optical Society of America.

An ideal polarizing beam splitter ${ }^{1,2}$ (PBS) is a device that separates two cotraveling orthogonally linearly polarized components of an incident light beam (usually denoted by $p$ and $s$ ) into two beams that propagate in different directions, with each split beam being purely polarized in one of the original orthogonal linear states. Practical PBSs are based on the phenomenon of double refraction, crystal polarizers such as the Rochon and Wollaston prisms, ${ }^{1}$ or interference in a multilayer coating embedded inside a (glass) cube. ${ }^{1,2}$

The objective of this paper is to describe a simple alternative PBS (Fig. 1) that consists of a plane-parallel optically isotropic dielectric slab of refractive index $n_{2}$ that is as low as possible, which is coated on both sides by a transparent thin film of refractive index $n_{1}$ that is as high as possible. The slab is oriented so that light is incident (from air, $n_{0}=1$ ) at an angle $\phi_{p}=\arcsin \left(u^{1 / 2}\right)$, where $u$ is the root between 0 and 1 of the quadratic equation ${ }^{3}$

$$
\begin{aligned}
a u^{2}+b u+c & =0, \\
a=n_{1}^{8}-n_{2}^{4}, \quad b & =2 n_{1}^{2} n_{2}^{4}-n_{1}^{8}\left(n_{2}^{2}+1\right), \\
c & =n_{1}^{4} n_{2}^{2}\left(n_{1}^{4}-n_{2}^{2}\right) .
\end{aligned}
$$

At $\phi_{p}$ the $p$-polarized component (parallel to the plane of incidence) is totally transmitted $\left(R_{p}=0, T_{p}=1\right)$ when the thickness of the film equals

$$
d_{p}=(\lambda / 4)\left(n_{1}^{2}-u\right)^{-1 / 2}
$$

or an odd multiple thereof. To achieve an acceptable PBS the $s$ component must be nearly totally reflected $\left(R_{s} \approx 1\right.$ and $T_{s} \approx 0$ ). The intensity reflectance for the $s$ polarization of one coated surface takes the simple form

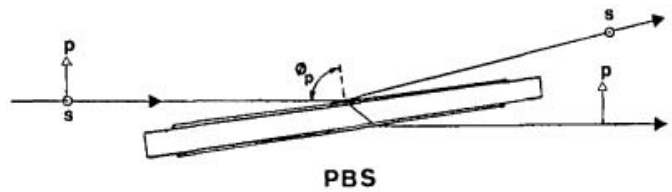

Fig. 1. Polarizing beam splitter using a coated dielectric slab. A very small $s$ component in the transmitted beam is not indicated. 


$$
R_{s}=\left[\left(n_{1}^{4}-n_{2}^{2}\right) /\left(n_{1}^{4}+n_{2}^{2}\right)\right]^{2} .
$$

About the best that one can do in the visible spectrum is to have $n_{1} \approx 2.5$ (e.g., $\mathrm{TiO}_{2}$ film) and $n_{2} \approx 1.4$ (substrate of a fluoride material). In this case Eq. (4) gives $R_{s}=0.818$, which is not high enough. However, two or more such coated slabs placed in parallel and in succession make a useful transmission polarizer. ${ }^{4,5}$

The situation is significantly favorably different in the IR where semiconductors become transparent and present an excellent choice of high-index thin-film coating material. For example, Ge has a refractive index of 4 (a higher value is possible depending on evaporation conditions) over a wide (3-13- $\mu \mathrm{m})$ IR spectral range. Lower-index substrates are also available in the IR, such as Irtran 1 and Irtran 3 (hotpressed polycrystalline compacts of $\mathrm{MgF}_{2}$ and $\mathrm{CaF}_{2}$, respectively) ${ }^{6}$ with $n_{2}<1.4$ for $\lambda>5 \mu \mathrm{m}$.

As a first specific example let us take a Ge/Irtran 3 filmsubstrate system at wavelength $\lambda=10.6 \mu \mathrm{m}$ of the $\mathrm{CO}_{2}$ laser. From Ref. 6 we find that $n_{1}=4(\mathrm{Ge})$ and $n_{2}=1.28$ (Irtran 3) at this wavelength. From Eqs. (1) and (2) we get $u=0.98585$, $\phi=83.17^{\circ}$. From Eq. (3) $d_{p}=683.90 \mathrm{~nm}$, and Eq. (4) gives $R_{s}=0.9747$. This reflectance is high enough to make this Ge-coated Irtran 3 slab a simple useful (although not ideal) PBS. The reflected light is, of course, totally $s$ polarized. The transmitted light is dominantly $p$ polarized with a small residual $s$ component. The extinction ratio $E R$ in transmission is given by

$$
\begin{aligned}
E R_{t} & =T_{s} / T_{p} \\
& =\left(1-R_{s}\right)^{2} \\
& =6.4 \times 10^{-4} .
\end{aligned}
$$

The degree of polarization $P$ of transmitted light for incident unpolarized light is given by

$$
\begin{aligned}
P_{t} & =\left(T_{p}-T_{s}\right) /\left(T_{p}+T_{s}\right) \\
& =0.9987,
\end{aligned}
$$

which confirms that the transmitted radiation is indeed almost totally $p$ polarized.

Performance approaching even closer the ideal PBS is attained if IR-transparent $(4-20-\mu \mathrm{m}) \mathrm{PbTe}$ with refractive index $n_{1}=5.1$ is used as the film material. ${ }^{7}$ Assuming $n_{2}=$ 1.28 as before (Irtran 3 slab, $\lambda=10.6 \mu \mathrm{m}$ ), we get $R_{s}=0.9904$; this impressively high reflectance falls short of the ideal by $<1 \%$. From Eqs. (5) and (6) we calculate $E R_{t}=10^{-4}$ and $P_{t}=$ 0.9998 . Because the reflection is perfectly polarizing, $E R_{r}=$ 0 and $P_{r}=1$. These results clearly demonstrate that this PBS is very practical. For completeness, we indicate that $\phi_{p}$ $=85.67^{\circ}$ and $d_{p}=529.83 \mathrm{~nm}($ at $\lambda=10.6 \mu \mathrm{m}$ ), conditions required to make the $\mathrm{PbTe}$-coated Irtran 3 slab function as PBS.

Angular separation between the split beams reflected from and transmitted by the coated-slab PBS is $180^{\circ}-2 \phi=13.66$ and $8.66^{\circ}$ for the $\mathrm{Ge}$ - and $\mathrm{PbTe}$-coated Irtran 3 devices, respectively. These separations are adequate and compare favorably with those obtained by the commonly used Rochon and Wollaston prisms. ${ }^{1}$

Parasitic beams resulting from multiple reflections within the slab are too weak to present a problem. The ratio of intensity of the second reflected beam (which is also totally $s$ polarized) to that of the primary reflected beam is equal to the transmission extinction ratio $E R_{t}$. If necessary, these feeble higher-order beams can be simply blocked off (spatially filtered) when the slab is sufficiently thick or slightly wedged.

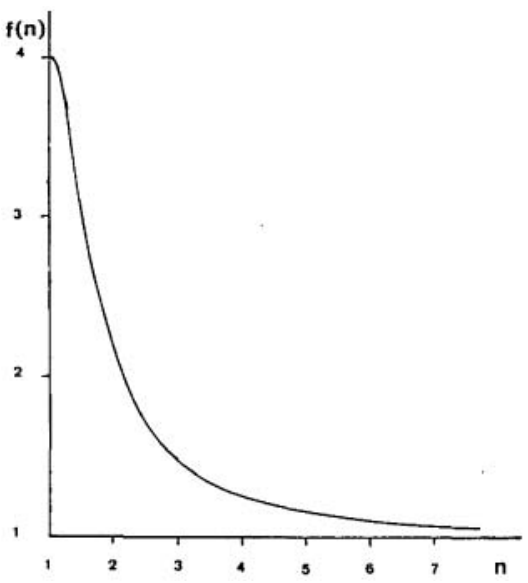

Fig. 2. Enhancement factor $f(n)$, Eq. (10), of the $s$ reflectance as a result of interference in an unbacked film of refractive index $n$. The layer is assumed to be of quarterwave optical thickness at the Brewster angle of incidence, $\phi_{\mathrm{B}}=\tan ^{-1} n$.

An interesting limiting case that leads to even higher performance is obtained when $n_{2}$ is assigned the least possible value of 1 . In this situation the PBS consists of two unbacked films (or sheets) each of quarterwave optical thickness which are separated by a uniform air gap. For simplicity we write $n_{1}=n$, since only one material is now involved. $\phi_{p}$ reverts back to the Brewster angle of the film, i.e., $\phi_{p}=\phi_{\mathrm{B}}$ $=\tan ^{-1} n\left[75.96\right.$ and $78.91^{\circ}$ for $n=4(\mathrm{Ge})$ and $5.1(\mathrm{PbTe})$, respectively]. From Eq. (4), the $s$ reflectance of the unbacked film at $\phi_{\mathrm{B}}$ is obtained by setting $n_{1}=n$ and $n_{2}=1$ :

$$
R_{s}=\left[\left(n^{4}-1\right) /\left(n^{4}+1\right)\right]^{2} \text {. }
$$

For a semi-infinite phase of film material

$$
\bar{R}_{s}=\left[\left(n^{2}-1\right) /\left(n^{2}+1\right)\right]^{2}
$$

at $\phi_{\mathrm{B}}$. From Eqs. (7) and (8), we may write

$$
R_{s}=f(n) \bar{R}_{s}, \quad \text {. }
$$

where

$$
f(n)=\left(n^{2}+1\right)^{4} /\left(n^{4}+1\right)^{2} .
$$

$f(n)$ represents the enhancement factor of the $s$ reflectance due to interference in the quarterwave film. Figure 2 shows that $f(n)$ falls monotonically from a maximum of 4 at $n=1$ (or infinitesimally $>1$ ) to 1 as $n \rightarrow \infty$.

Returning to Eq. (7) we get $R_{s}=0.9845$ when $n=4$. [For this $n, \bar{R}_{s}=0.7786$ and $f(4)=1.26453$.] For a two-film PBS, $E R_{t}=2.4 \times 10^{-4}$ and $P_{t}=0.9995$. When $n=5.1$, we have $R_{s}$ $=0.9941, E R_{t}=3.47 \times 10^{-5}$, and $P_{t}=0.9999$. Together with $E R_{r}=0$ and $P_{r}=1$, these results indicate truly outstanding performance by a simple system of two unbacked films. Even with one unbacked high-index film $(n>5)$, a PBS with acceptable characteristics is obtained.

A finding that adds to the importance of PBS using unbacked films is that performance is essentially achromatic over at least one spectral octave (neglecting material dispersion) and is only slightly affected by large errors of thickness around the quarterwave condition. A drastic change would be to reduce the normalized thickness by half. For an unbacked film of one (three or any odd multiple of) eighth-wave optical thickness, it can be proved that the $s$ reflectance at the Brewster angle $\left(R_{p}=0\right)$ is given by

$$
R_{s}=\frac{n^{8}-2 n^{4}+1}{n^{8}+6 n^{4}+1} .
$$


When $n=4$, Eq. (11) gives $R_{s}=0.9695$. Compared with $R_{s}=$ 0.9845 for the quarterwave film, the one-half reduction of film thickness (or the equivalent doubling of wavelength) lowers the reflectance by only 0.015 ! For a pair of eighthwave unbacked films of $n=4$, we calculate $E R_{t}=9.3 \times 10^{-4}$ and $P_{t}=0.9981$.

PBS using unbacked high-index quarterwave (or nearquarterwave) films is expected to prove especially useful for the now important far-IR and millimetric waves. The reason is obvious: unbacked films will require no special art to make. One will need only look for transparent materials of the highest possible refractive index.

The kind hospitality of the Département de Physique du Solide, Université de Provence, where this work was done, and a Fulbright Senior Research Scholar Award are gratefully acknowledged.

\section{References}

1. J. M. Bennett and H. E. Bennet', "Polarization," in Handbook of Optics, W. G. Driscoll and W. Vaughan, Eds. (McGraw-Hill), New York, 1978), Sec. 10.

2. J.A. Dobrowolski, "Coatings and Filters," in Handbook of Optics, W. G. Driscoll and W. Vaughan, Eds. (McGraw-Hill, New York, 1978), Sec. 8.

3. O. S. Heavens, Optical Properties of Thin Solid Films (Butterworths, London, 1955), p. 239.

4. Ref. 1, p. 10-100.

5. Ref. 2, p. 8-74.

6. W. L. Wolfe, "Properties of Optica! Materials," in Handbook of Optics, W. G. Driscoll and W. Vaughan, Eds. (McGraw-Hill, New York, 1978), Sec. 7, p. 7-93.

7. Ref. 6, p. 7-111. input fiber is coupled to two output fibers via a graded-index lens and a mirror which tilts as a function of acceleration. To increase sensitivity a stripe pattern is placed over the end of the fibers.

G.L.M.

4,596,467

24 June 1986 (Cl. 356-363)

Dissimilar superimposed grating precision alignment and gap measurement systems.

J. L. BARTELT. Assigned to Hughes Aircraft Co. Filed 16 Mar. 1984.

Precision superposition of a mask and a substrate used to make integrated circuits, for example, is obtained with diffraction gratings of differing spacing. Diffraction order intensity is said to be very sensitive to alignment and spacing
of the gratings.

$4,600,304$

15 July 1986 (Cl. 356-138)

Optical level.

O. KAFRI and A. LIVNAT. Assigned to The State of Israel, Atomic Energy Commission. Filed 2 Feb. 1983.

Reflected light from a nominally horizontal object surface and from the surface of a liquid pool supported by the object is used to make two sets of moire fringes. Misalignment of these fringes determines the object level with
respect to gravity. The method is more sensitive than a spirit level but less sensitive than interferometry to small movements of the object. C.F.M.

$4,601,533$

22 July 1986 (Cl. 350-3.7)

Laser eye protection visor using multiple holograms.

G. E. MOSS. Assigned to U.S.A. as represented by Secretary of the Navy. Filed 29 Oct. 1982.

Highly selective holographic reflectors on transparent visors are proposed for eye protection from laser radiation while allowing clear broad-spectrum vision. 